\title{
On vortex motions in air
}

\section{G. Quincke}

To cite this article: G. Quincke (1894) On vortex motions in air, Philosophical Magazine Series 5, 38:230, 159-160, DOI: $10.1080 / 14786449408620615$

To link to this article: http://dx.doi.org/10.1080/14786449408620615

$$
\text { 曲 Published online: } 08 \text { May } 2009 .
$$

Submit your article to this journal

Џ Article views: 2

Q View related articles $\asymp$ 
If the polarizing apparatus is turned while at the same time the strength of the current is measured at the galvanometer, two maxima and two minima are observed in the course of a single rotation. The minima occur when the plane of polarization of the light is parallel to the plane of incidence of the rays on the kathode; the maxima are in positions at an angle of $90^{\circ}$ with them. The ratio of maxima to minima is about $10: 1$. If while the plane of polarization is parallel to the plane of incidence, a quarta plate about 2 millim. thick, cut at right angles to the optical axis, is placed in the path of the polarized light, the current increases about sevenfold, corresponding with the rotation of the plane of polarization due to the quartz. When the plane of polarization is at right angles to the plane of incidence, a quartz plate has the opposite effect; the strength of the current diminishes, as was to be expected, in a corresponding ratio. Apart from a slight enfeeblement of the current due to the loss of light caused by its interposition, a clear glass plate has no effect in either position.

According to the investigations of MM. Trouton ('Nature,' vol. xxxix. p. 393), Klemencic (Wiedemann's Annalen, vol. xlv, p. 77), and Righi (Rend. della R. Ac. dei Lincei, vol. xi. p. 161) it must be taken for granted that, in Hertz's rays of electrical force, the plane of polarization is at right angles to the direction of the electrical displacement. If the motion in the light rays is regarded as analogous, the result of the experiments described would be thus expressed.

The luminous electrical current attains its maximum when the electrical displacements in the luminous ray take place in the plane of incidence, its minimum when they are at right angles thereto. In the former case the electrical vibrations contain a component normal to the kathode, but not in the second. We might be tempted to seek in these changes of potential normal to the kathode, and induced by the electrical rays, the force which impels the negative electricity to leave the kathode. Whether this suggestion is correct, can perhaps be ascertained by further experiments on the dependence of the luminous electrical action on the angle of incidence of the polarized light, and their connexion with the quantities of light reflected from and retained by the kathode.Berliner Berichte, February 8, 1894. (Communicated by the Authors.)

ON VORTEX MOTIONS IN AIR. BY G. QUINCKE.

At the meeting of the Natural History and Medical Society of Feb. 7, 1890, I discussed the motion of falling spheroids of oil in water, the specific gravity of which was increased somewhat by the addition of chloroform. Such a spheroid falls vertically in water at rest. But if two spheroids of oil fall simultaneously close to each other, they approach and recede from each other in falling. The path and the time of fall depend on the distance apart and the velocity of the falling spheroids. This peculiar. motion is caused by the vortices which are produced by the falling spheroids of oil in the water about them, which had hitherto 
been at rest. The particles of water in the plane of symmetry between the falling spheroids remain at rest.

Instead of two spheres, one may be allowed to fall near a plane vertical wall, which then acts as plane of symmetry. The spheroid falls as it were with its image in the plane vertical wall, and approaches and recedes from this.

These experiments with heavy oil spheroids in water are tedious, and can only be shown to a small audience.

Analogous phenomena may be produced before a larger circle of hearers by allowing two soap bubbles filled with coal-gas to ascend near each other, or a single bubble near a vertical wall.

In ascending, the distance of the two soap bubbles from each other, or of a single one from its image in the vertical wall, is first smaller and then greater, and the cause again is the rortex movements in the air due to the ascending soap bubbles.

In order to fill two soap bubbles simultaneously with coal-gas they are produced at the ends of a T-piece of glass, blown out in the form of horizontal cups, to which the gas passes from the centre tube through a T-shaped perforated glass stopper.

Similar phenomena occur when small dust particles fall in air or liquid at rest, or if a current of air or liquid strikes against particles of dust at rest. The motion of the small particles is influenced by the presence and form of the solid wall in the vicinity.-Wiedemann's Annalen, July 1894.

ON A NEW APPARATUS FOR THE PRODUCTION OF HIGH PRESSURE. BY PROF. S. W. STRATTON*.

Not long since, while designing a piece of apparatus for the production of high pressure, it occurred to me that many of the difficulties encountered in the measurement of such pressures might be avoided by the employment of several short mercurycolumns connected in series by means of a less dense liquid, as shown in the accompanying sketch.

Such a gauge would possess all the advantages of the ordinary mercury-column, and be within the limits of space to be had in the laboratory. For many purposes the last tube only need be made of glass, and the scale reduced accordingly.

Thinking that this principle may be new, and of value to some who are employing high pressures in the laboratory, it is submitted for publication.

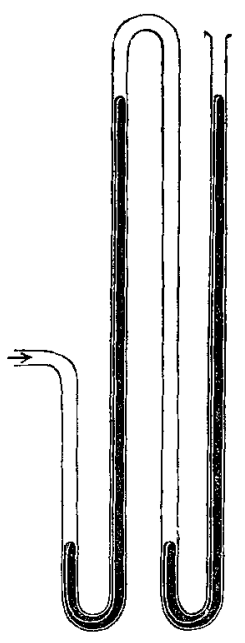

The Ryerson Physical Laboratory,

University of Chicago,

May 21, 1894.

* Communicated by Prof. A. A. Michelson. 